J. Clin. Chem. Clin. Biochem.

Vol. 21, 1983, pp. 805-811

\title{
Bestimmung von $\beta$-Rezeptoren an polymorphkernigen intakten Leukocyten im autologen Plasma $^{1}$ )
}

\author{
Von M. Lehmann, P. Schmid, E. Bergdolt \\ Medizinische Universitätsklinik, Abt. Sport- und Leistungsmedizin (Ärztlicher Direktor: Prof. Dr. J. Keul) \\ Freiburg/Breisgau
}

\section{H. Porzig}

Pharmakologisches Institut der Universität Bern und

\section{J. Keul}

Medizinische Universitätsklinik, Abt. Sport- und Leistungsmedizin (Ärztlicher Direktor: Prof. Dr. J. Keul) Freiburg/Breisgau

(Eingegangen am 18. März/22. Juli 1983)

Zusammenfassung: Zur Bestimmung der $\beta$-Rezeptoren-Dichte wurde die spezifische Bindung von $\left[{ }^{3} \mathrm{H}\right] \mathrm{Dihy}-$ droalprenolol an intakten polymorphkernigen Leukocyten bei 6 ausdauertrainierten $\left(\mathrm{VO}_{2} \max .65,7 \pm 2,0\right.$ $\mathrm{ml} / \mathrm{kg} \cdot \min )$ und 9 nicht ausdauertrainierten männlichen Probanden $\left(\mathrm{VO}_{2} \max .52,0 \pm 4,0 \mathrm{ml} / \mathrm{kg} \cdot \min \right)$ untersucht. Die spezifische Bindung ist definiert als Differenz zwischen Gesamtbindung und nicht verdrängbarer unspezifischer Bindung. Für die Bindungsstudien wurden die Leukocyten im autologen Plasma reinkubiert. Die spezifische Bindung zeigt bei trainierten und untrainierten Probanden ein Sättigungsverhalten bei ungefähr̈ $2 \mathrm{nmol} / /$ Dihydroalprenolol. Sie beträgt $85 \%$ (0,1 nmol/1 Dihydroalprenolol) bis $51 \%(2,0 \mathrm{nmol} / 1$ Dihydroalprenolol) der Gesamtbindung. $B_{\max }$. beträgt, basierend auf der Scatchard-Analyse, 41,2 fmol/10 Zellen (Trainierte) und $21,6 \mathrm{fmol} / 10^{7}$ Zellen (Untrainierte). Die Dissonanzkonstante $\left(\mathrm{K}_{\mathrm{D}}\right)$ wurde zu 0,44 nmol// Dihydroalprenolol (Untrainierte) und 0,49 nmol/I Dihydroalprenolol (Trainierte) berechnet, die Zahl der $\beta$-adrenergen Bindungsstellen beträgt ungefähr 1300 (Untrainierte) und 2500 pro Zelle (Trainierte). Die spezifische Bindung von Dihydroalprenolol an intakte polymorphkernige Leukocyten von ausdauertrainierten ist signifikant höher als bei untrainierten Probanden. Die trainings-abhängige Änderung der $\beta$-Rezeptoren-Dichte kann ein Indikator einer gesteigerten Empfindlichkeit gegenüber Katecholaminen sein.

\section{Determination of $\beta$-receptors on live human polymorphonuclear leukocytes in autologous plasma}

Summary: The binding of tritium labelled radioligand dihydroalprenolol was investigated on live polymorphonuclear leukocytes of 6 endurance trained $\left(\mathrm{VO}_{2} \max .65 .7 \pm 2.0 \mathrm{ml} / \mathrm{kg} \cdot \mathrm{min}\right)$, and 9 non-endurance trained subjects $\left(\mathrm{VO}_{2} \max .52 .0 \pm 4.0 \mathrm{ml} / \mathrm{kg} \cdot \mathrm{min}\right)$. The specific binding of dihydroalprenolol is seen as an indicator of the $\beta$-receptor density. The specific binding of dihydroalprenolol is defined as the difference between the total binding and that amount of dihydroalprenolol that could not be displaced. The leukocytes were reincubated for the binding studies in their autologous plasma. The specific binding of dihydroalprenolol on live polymorphonuclear leukocytes shows a levelling off behaviour at a concentration of approximately 2 $\mathrm{nmol} / \mathrm{l}$ dihydroalprenolol in trained as well as in untrained subjects. The specific binding amounts to about $85 \%(0.1 \mathrm{nmol} / 1$ dihydroalprenolol $)$ to $51 \%(2.0 \mathrm{nmol} / \mathrm{l}$ dihydroalprenolol $)$ of the total binding. Based on Scatchard analysis, $B_{\max }$ was determined as $41.2 \mathrm{fmol} / 10^{7}$ cells (trained subjects), and $21.6 \mathrm{fmol} / 10^{7}$ cells

1) Mit Unterstützung des Bundesinstitutes für Sportwissenschaft, Köln-Lövenich. 
(untrained subjects). $\mathrm{K}_{\mathrm{D}}$ is $0.44 \mathrm{nmol} / \mathrm{l}$ dihydroalprenolol (untrained subjects), and $0.49 \mathrm{nmol} / 1$ dihydroalprenolol (trained subjects). The $\beta$-adrenergic binding sites are approximately 1300 (untrained subjects), and 2500 bindings sites/cell (trained subjects). The specific binding of dihydroalprenolol on live polymorphonuclear leukocytes is significantly higher in trained than in untrained subjects. This training dependent change in $\beta$-receptor density may be an indicator of an increased sensitivity to catecholamines.

\section{Einführung}

Das Verhalten $\beta$-adrenerger Bindungsstellen kann durch Untersuchungen mit Radioliganden an Membranpräparaten und intakten Zellen beobachtet werden (1). Diese Studien beruhen auf der hohen Affinität von $\beta$-Sympatholytica zu $\beta$-Rezeptoren. Schwierigkeiten bereitet das $A$ usma $\beta$ der unspezifischen Bindung oder lysosomalen Aufnahme des Radioliganden durch intakte Zellen. Fortschritte wurden durch Hemmung der lysosomalen Aufnahme $\beta$ adrenerger Liganden durch Chloroquin (2) oder Phentolamin (3) erzielt. In Vorversuchen bemerkten wir eine deutliche Aggregationsneigung der Zellen nach Inkubation in der von Dulis \& Wilson angegebenen Pufferlösung (2). Mit der vorliegenden Arbeit wird deshalb das Verhalten der $\beta$-Rezeptoren an intakten polymorphkernigen Leukocyten im autologen Plasma bei ausdauer- und nicht ausdauer-trainierten Probanden untersucht.

\section{Methodik}

Probanden

Untersucht wurden 15 gesunde, männliche Probanden, von denen 6 ein regelmäßiges körperliches, dynamisches Ausdauertraining von mehr als 4-6 Stunden pro Woche betreiben (Tab. 1).

Tab. 1. Anthropometrische Daten und maximale Sauerstoffaufnahmefähigkeit der untersuchten Probanden.

\begin{tabular}{|c|c|c|c|c|c|c|}
\hline & $\mathrm{n}$ & & $\begin{array}{l}\text { Alter } \\
\text { (Jahre) }\end{array}$ & $\begin{array}{l}\text { Größe } \\
(\mathrm{cm})\end{array}$ & $\begin{array}{l}\mathrm{Ge}- \\
\text { wicht } \\
(\mathrm{kg})\end{array}$ & $\begin{array}{l}\mathrm{VO}_{2} \\
\mathrm{max} . \\
(\mathrm{ml} / \mathrm{kg} \\
\cdot \mathrm{min})\end{array}$ \\
\hline $\begin{array}{l}\text { Ausdauer- } \\
\text { trainierte }\end{array}$ & 6 & $\begin{array}{l}\overline{\mathbf{x}} \\
\mathrm{S}\end{array}$ & $\begin{array}{r}32 \\
5\end{array}$ & $\begin{array}{r}179 \\
9\end{array}$ & $\begin{array}{r}68 \\
5\end{array}$ & $\begin{array}{r}65,7 \\
2,0\end{array}$ \\
\hline $\begin{array}{l}\text { Nicht- } \\
\text { Ausdauer- } \\
\text { trainierte }\end{array}$ & 9 & $\begin{array}{l}\bar{x} \\
S\end{array}$ & $\begin{array}{r}28 \\
3\end{array}$ & $\begin{array}{r}181 \\
5\end{array}$ & $\begin{array}{r}71 \\
9\end{array}$ & $\begin{array}{r}52,0 \\
4,0\end{array}$ \\
\hline
\end{tabular}

* maximale Sauerstoffaufnahmefähigkeit als Bruttokriterium der maximalen aeroben Kapazität und dynamischen Leistungsfähigkeit, bestimmt während stufenweiser Laufbandergometrie.

\section{Substanzen}

Isolierung der polymorphkernigen Lèukocyten: Ficoll-Paque wird von Deutsche Pharmacia, Freiburg, FRG, bezogen, Heparin (Liquemin) in der Konzentration von $5000 \mathrm{E} / \mathrm{ml}$ vön Hoffmann-LaRoche, Grenzach, FRG, Plasmasteril ${ }^{\circledR}$ von Fresenius, Bad Homburg, FRG. Die $1 \mathrm{~mol} / \mathrm{l}$ Hepes-Pufferlösung wird von Gibco Europe, Karlsruhe, FRG, physiologische Kochsalżlösūng von Boehringer Mannheim, Mannheim, FRG, Dextrạn T 500 von Roth, Karlsruhe, FRG, geliefert.

Bindungsstudie: Hieržu wer̀den benötigt: $\mathrm{MgCl}_{2} \cdot 6 \mathrm{H}_{2} \mathrm{O}$ (Merck, Darmstadt, FRG), EDTA (Fluka, Buchs, CH), Chloroquine-diphosphat (Sigma, St. Louis, U.S.A.), $\mathrm{HCl}, \mathrm{NaOH}$ und Eisessig; ferner 1 molh Hepes-Pufferlösuñg (s.o:), Hariks-buffered-saltsolution, phenolrot-, calcium- und magnesiumfrei (Gibco Europe, Karlsruhe, FRG), $\left[{ }^{3} \mathrm{H}\right](-)$ Dihydroalprenolol-hydrochlorid, spezifische Aktivität ungefähr $3330 \mathrm{TBg} / \overline{\mathrm{mol}}(90 \mathrm{Ci} / \mathrm{mmol})(\mathrm{NEN}$, Dreieich, FRG), (-)Timolol-maleat (MSD Sharp ự Dohme, München, FRG), Whatmann Filter GF/F, $25 \mathrm{~mm} \varnothing$ (Bender und Hobein, Freiburg, FRG), Toluol (Merck, Darmstädt, FRG) und Butyl-PBD (Packard, Zürich, $\mathrm{CH}$ ). Protosol wurde von NEN, Dreieich, FRG, bezogen.

\section{Versuchsablauf}

Die Bindungsstudien wurden an polymorphkernigen Leukocy.ten durchgeführt. Der Versuchsablauf gliedert sich in Isolierung der Leukocyten (Dichte-Gradienten-Zentrifugation und Sedimeñntation) und Bindungsstudie (Inkubation, Filtervorgang und Ermitteln der gebundenen Aktivität an $\left[{ }^{3} \mathrm{H}\right]$ Dihydroalprenolol). $50 \mathrm{ml}$ Blut (Heparin: $10 \mathrm{E} / \mathrm{ml}$ ) wurden zwischen 8 und $8.30 \mathrm{Uhr}$ abgenommen und sofort weiterbearbeitet. Die Dichte-GradientenZentrifugation wurde nach Böyum (4) mit Ficoll-Paque (400 g, 40 min, $20^{\circ} \mathrm{C}$ ) durchgeführt. Das Vollblut wurde hierzu im Verhältnis 1:4 mit physiol. NaCl-Lösung gemischt und $16 \mathrm{ml}$ auf $6 \mathrm{ml}$ Ficoll=Paque überschichtet. Die Bodenphase, bestehend aus polymorphkernigen Leukocyten und Erythrocyten wurde anschlieBend mit Plasmasteril im Verhältnis $1+1$ gemischt und etwa 75 min bei $1 \mathrm{~g}$ einer Sedimentation unterzogen. Das Plasmasteril war hierfür mit Hepes-Pufferlösung (12,5 ml 1 mol/1. Hepes-Lösung + $487,5 \mathrm{ml}$ Plasmasteril ${ }^{\circledR}$, entsprechend einer $25 \mathrm{mmol} / \mathrm{i}$ Hepes-Lösung) und Heparin ( $5 \mathrm{E} / \mathrm{ml})$ auf $\mathrm{pH} \mathrm{7,4} \mathrm{eingestellt} \mathrm{worden.} \mathrm{(1}$ mol/1 Hepes-Puffer: 23,83 g 2-[4-(2-Hydroxyäthyl)-piperazinyl(1)]-ethansulfonsäure ad $100 \mathrm{ml}$ bidest. Wasser nach Ëinstellen und Kontrolle des pH-Wertes mit $\mathrm{NaOH}$ ). Die Sedimentation wưrde nach Böyum (4) durch Zugabe von Dextran verbessert. Nach der Dichte-Gradienten-Zentrifugation war die obere Fraktion, bestehend aus einem Plasma-NaCl-Gemisch (1:4) zur weiteren Verwendung separiert worden. Die isolierten polymorphkernigen Leukocyten wurden dreimal im autologen Plasma- $\mathrm{NaCl}$ Gemisch gewaschen und auf ein Volumen von 2,4 ml Zellsuspension eingestellt. Die Konzentration an Leukocyten dieser Suspension betrug $16,7 \pm 7,7 \times 10^{9} /(\overline{\mathrm{x}} \pm \mathrm{s})$. Die Bestimmung der $\beta$ - $R e$ zeptoren an den intakten polymorphkernigen Leukocyten erfolge nach Dulis \& Wilson (2), Baer \& Porzig (5) bzw. Porzig et al. (6). In Abänderung dieser Methoden erfolgte die Inkubation der Zellen im autologen Plasma, 1:4 mit physiol. Nacl-Lösung verdünnt. Entgegen dem Vorschlag von Dulis \& Wilson (2) wurde die Verdrạngung des $\left[{ }^{3} \mathrm{H}\right]$ Dihydroalprenolols aus seiner spezifischen Bindung nicht durch Propranolol, sondern wie von Porzig et al. (6) empfohlen, mittels (-)Timolol vorgenommen: $200 \mu$ der Zellsus- 
pension wurden inkubiert in $2000 \mu l$ der 1:4 autologen Plasma$\mathrm{NaCl}-\mathrm{Verdünnung}$, und zwar einmal mit $10^{-6} \mathrm{~mol} /(-)$ Timolol und ein zweites Mal ohne Timolol-Zusatz. Ad $50 \mathrm{ml}$ Plasma waren zuvor $5 \mathrm{mg} \mathrm{MgCl} \cdot 6 \mathrm{H}_{2} \mathrm{O}, 1,29 \mathrm{mg}$ Chloroquin ( $=50 \mu \mathrm{mol} / \mathrm{l}$ ) und $18,5 \mathrm{mg}$ EDTA gegeben und der pH-Wert mit $1 \mathrm{~mol} / \mathrm{NaOH}$ auf 7,4 eingestellt worden. Die Bindungsstudie begann durch $\mathrm{Zu}-$ gabe von $40 \mu$ l Dihydroalprenolol der Konzentration 0,1, 0,5, 1,0, 2,0 und $5.0 \mathrm{nmol} /$. Inkubationslösung und Dihydroalprenolol enthaltende Zählgefäße wurden im Abstand von 2,5 min ins Schüttelwasserbad $\left(37^{\circ} \mathrm{C}\right)$ gegeben, nach $5 \mathrm{~min}$ Äquilibrierung erfolgte die Zugabe von $200 \mu \mathrm{l}$ Zellsuspension und nach weiteren 25 min Inkubation wurden die Proben im Abstand von je 2,5 min filtriert. Hierzu wurden im Duplikat $1000 \mu \mathrm{l}$ Inkubationsmischung auf je 1 Filter gegeben, (die Filterkammer enthielt dabei bereits $7 \mathrm{ml}$ eiskalte Waschlösung), sofort filtriert und noch zweimal mit wiederum $7 \mathrm{ml} \mathrm{Waschlösung} \mathrm{nachgespült.} \mathrm{Diese} \mathrm{drei} \mathrm{Ar}$ beitsschritte nahmen jeweils ungefähr $4 \mathrm{~s}$ in Anspruch. Als Waschlösung diente eine calcium- und magnesiumfreie HepesHanks-Salt-Solution, zu der $\mathrm{MgCl}_{2}$, EDTA und Hepes-Puffer in gleicher Konzentration wie zur Inkubationslösung hinzugefügt wurden. (Hepes-Hanks-Salt-Solution: $136,8 \mathrm{mmol} / / \mathrm{NaCl}, 5,3$ $\mathrm{mmol} / \mathrm{KCl}, 0,26 \mathrm{mmol} / \mathrm{Na} \mathrm{NaPO}_{4}, 4,1 \mathrm{mmol} / \mathrm{NaHCO} 3,0,31$ $\mathrm{mmol} / \mathrm{KH}{ }_{2} \mathrm{PO}_{4}, 5,5 \mathrm{mmo} / \mathrm{G}$ Glucose). Die Konzentration der Zellen pro Filter betrug $1490 \pm 680 \times 10^{3}(\overline{\mathrm{x}} \pm \mathrm{s})$. Die Filter wurden sofort in ein $20 \mathrm{ml}$ Zählgefäß gegeben und jeweils mit 0,6 $\mathrm{ml}$ Protosol-Ethanol-Gemisch $(1+1)$ überschichtet. Am folgenden Morgen wurden $10 \mu \mathrm{l}$ Eisessig und $10 \mathrm{ml}$ Toluol hinzugegeben, das $4 \mathrm{~g} / 1$ Butyl-PBD ((2-(4-tert-Butylphenyl)-5-(4-biphenylyl)-1,3,4-oxadiazol)) enthielt. Anschließen wurde die Aktivität an Tritium gemessen (Packard Liquid-Scintillation-System TRICARB 300).

\section{Auswertung der Untersuchungsresultate}

In den Tabellen wurden Median, 50\% Vertrauensbereich und Gesamtbereich angegeben, ausgenommen Tabelle 1, und in den Abbildungen 1 und 2 der Medianwert graphisch dargestellt. Die statistische Untersuchung beruht auf dem Wilcoxon-Test für un- verbundene Stichproben (Rangordnungstest), die ScatchardFunktion wurde nach dem Prinzip der kleinsten Summe der Abweichungsquadrate berechnet (Signifikanzniveau $p<0,05$ ).

\section{Ergebnisse}

Als spezifisch gebundene Menge an Dihydroalprenolol wird die Differenz zwischen Gesamtbindung und unspezifischer Bindung gewertet. Die unspezifische Bindung wurde mittels der lysosomotropen Substanz Chloroquin in Úbereinstimmung mit Dulis \& Wilson (2) deutlich reduziert. Sie betrug zwischen $15 \%$ (bei 0,1 nmol/1 Dihydroalprenolol), $18 \%(0,5$ nmol/1 Dihydroalprenolol), 31\% (1,0 nmol/1 Dihydroalprenolol) und $49 \%$ ( $2 \mathrm{nmol} / \mathrm{l}$ Dihydroalprenolol) (Tab. 2) der Gesamtbindung oder -aufnahme. Entsprechend lag die spezifische Bindung von Dihydroalprenolol im Mittel (Median) bei $85(0,1 \mathrm{nmol} / 1$ Dihydroalprenolol) bis $51 \%$ (2 nmol/1 Dihydroalprenolol). In Abwesenheit von Chloroquin betrug die spezifische Bindung für Dihydroalprenolol bei 8 Vorversuchen, in Úbereinstimmung mit Dulis \& Wilson (2), zwischen $0-20 \%$ der Gesamtbindung, bzw. -aufnahme. Die spezifische Bindung von Dihydroalprenolol zeigt um 2 nmol/1 Dihydroalprenolol ein Sättigungsverhalten (Tab. 2, Abb. 1). Die Erhöhung der Konzentration auf $5 \mathrm{nmol} / 1$ Dihydroalprenolol erbringt keine weitere wesentliche $\mathrm{Zu}$ nahme der spezifischen Bindung.

Tab. 2. Spezifische und unspezifische Bindung von $\left[{ }^{3} \mathrm{H}\right]$ Dihydroalprenolol an polymorphkernige Leukocyten zur Charakterisierung der $\beta$-Rezeptoren bei ausdauertrainierten $(T ; n=6)$ und nicht ausdauertrainierten Probanden $(U ; n=9)$.

\begin{tabular}{|c|c|c|c|c|c|c|c|}
\hline \multirow{3}{*}{$\begin{array}{l}\text { Inkubation } \\
\text { an }\left[{ }^{3} \mathrm{H}\right] \mathrm{Di}- \\
\text { hydroal- } \\
\text { prenolol } \\
\text { (nmol/l) }\end{array}$} & & \multicolumn{2}{|c|}{ Spezifische Bindung } & \multicolumn{2}{|c|}{$\begin{array}{l}\text { Spezifische Bindung in \% } \\
\text { der Gesamtbindung }\end{array}$} & \multicolumn{2}{|c|}{ Unspezifische Bindung } \\
\hline & & \multicolumn{2}{|c|}{ (fmol/10 $10^{7}$ Zellen) } & \multicolumn{2}{|l|}{$(\%)$} & \multicolumn{2}{|c|}{ (fmol/107 Zellen) } \\
\hline & & U & $\overrightarrow{\mathrm{T}}$ & $\mathbf{U}$ & $\dot{T}$ & $U$ & $T$ \\
\hline 0,1 & $\begin{array}{l}\overline{\mathbf{x}} \\
\mathbf{5 0 \%} \\
\widetilde{\mathbf{R}}\end{array}$ & $\begin{array}{l}2,5 \\
1,0-2,7 \\
0,6-5,3\end{array}$ & $\begin{array}{l}4,0 \\
2,1-4,6 \\
1,3-5,9\end{array}$ & $\begin{array}{l}82 \\
68-87 \\
58-96\end{array}$ & $\begin{array}{l}84 \\
63-88 \\
47-95\end{array}$ & $\begin{array}{l}0,4 \\
0,2-0,8 \\
0,1-1,0\end{array}$ & $\begin{array}{ll}0,7 & \\
0,4- & 1,0 \\
0,2- & 6,6\end{array}$ \\
\hline 0,5 & $\begin{array}{l}\overline{\mathbf{x}} \\
50 \% \\
\overline{\mathbf{R}}\end{array}$ & $\begin{array}{l}8,1 \\
3,1-9,7 \\
1,9-13,5\end{array}$ & $\begin{array}{l}15,3 \\
14,2-19,0 \\
12,5-22,9\end{array}$ & $\begin{array}{l}76 \\
6 \dot{3}-85 \\
58-96\end{array}$ & $\begin{array}{l}82 \\
67-88 \\
61-93\end{array}$ & $\begin{array}{l}2,0 \\
1,0-3,4 \\
0,5-3,9\end{array}$ & $\begin{array}{l}2,6 \\
1,0-7,7 \\
1,0-12,9\end{array}$ \\
\hline 1,0 & $\begin{array}{l}\overline{\mathbf{x}} \\
50 \% \\
\mathbf{R}\end{array}$ & $\begin{array}{r}11,3 \\
10,4-19,4 \\
7,9-20,9\end{array}$ & $\begin{array}{l}22,7 \\
21,2-28,3 \\
20 ; 6-32,0\end{array}$ & $\begin{array}{l}63 \\
54-69 \\
22-78\end{array}$ & $\begin{array}{l}78 \\
63-80 \\
61-82\end{array}$ & $\begin{array}{l}6,5 \\
5,9-8,7 \\
3,5-10,7\end{array}$ & $\begin{array}{l}8,7 \\
5,5-12,5 \\
4,1-16,5\end{array}$ \\
\hline 2,0 & $\begin{array}{l}\bar{x} \\
50 \% \\
\mathbf{R}\end{array}$ & $\begin{array}{l}16,9 \\
13,2-22,0 \\
10,0-22,7\end{array}$ & $\begin{array}{l}30,6 \\
25,0-35,1 \\
23,6-35,7\end{array}$ & $\begin{array}{l}51 \\
44-57 \\
25-59\end{array}$ & $\begin{array}{l}54 \\
50-61 \\
38-64\end{array}$ & $\begin{array}{r}18,5 \\
14,1-22,6 \\
5,3-30,9\end{array}$ & $\begin{array}{l}25,0 \\
15,4-36,7 \\
12,5-39,3\end{array}$ \\
\hline 5,0 & $\begin{array}{l}\bar{x} \\
50 \% \\
R\end{array}$ & $\begin{array}{l}17,9 \\
14 ; 6-21,7 \\
12,1-30,2\end{array}$ & $\begin{array}{l}30,9 \\
28,2-36,3 \\
27,1-38,7\end{array}$ & $\begin{array}{l}27 \\
19-37 \\
13-59\end{array}$ & $\begin{array}{l}35 \\
30-37 \\
19-38\end{array}$ & $\begin{array}{r}54,4 \\
42,7-76,5 \\
7,3-91,9\end{array}$ & $\begin{array}{l}60,6 \\
50,7-81,7 \\
19,6-114,5\end{array}$ \\
\hline
\end{tabular}

$\overline{\mathbf{x}}=$ Median, $\quad 50 \%$ - Vertrauensbereich, $\quad \mathbf{R}=$ Gesamtbereich 
Die Unterteilung des Gesamtkollektivs in 6 ausdauertrainierte und 9 nicht-ausdauertrainierte Probanden zeigt, daß die spezifische Bindung von Dihydroalprenolol und somit die Rezeptorzahl trainingsabhängig ist (Tab. 2). Die spezifische Bindung von Dihydroalprenolol liegt bei den trainierten für die Konzentration 0,5 bis $5 \mathrm{nmol} / 1$ Dihydroalprenolol signifikant höher als bei den nicht trainierten Probanden (Abb. 1). Dies trifft nicht zu für die unspezifische Aufnahme von Dihydroalprenolol durch die Zellen. Wenngleich unspezifische Bindung (Aufnahme) von Dihydroalprenolol durch die polymorphkernigen Leukocyten der Trainierten, wie auch die spezifische Bindung in \% der Gesamtbindung im Mittel (Median) etwas höher sind als bei den Untrainierten (Tab. 2).

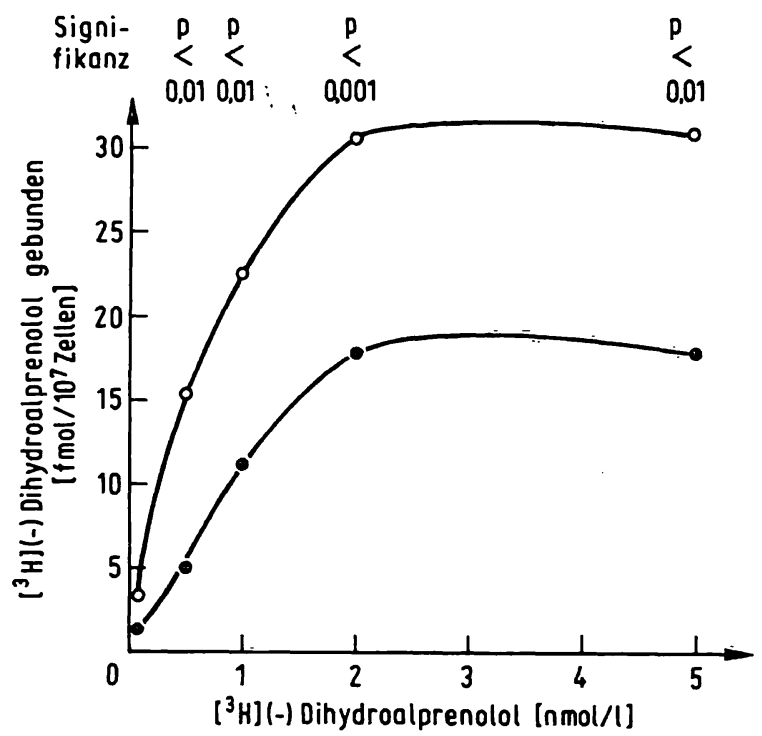

Abb. 1. Polymorphkernige Leukocyten ausdauertrainierter Probanden $(n=6,0-0)$ zeigen eine signifikant höhere Bindung an Dihydroalprenolol als Zellen von nicht ausdauertrainierten Personen $(n=9$, $0-0)$.

Mittels Gleichgewichtsdialyse (7) von physiologischer $\mathrm{NaCl}$-Lösung gegen das 1:4 autologe Plasma$\mathrm{NaCl}$-Inkubations-Medium - bei Zugabe von ED$\mathrm{TA}, \mathrm{MgCl}_{2}$ und Chloroquin zu beiden Lösungen ergab sich für den Konzentrationsbereich von 0,1 nmol/l-10 nmol/1 Dihydroalprenolol eine freie, nicht eiweißgebundene Dihydroalprenolol-Fraktion von 0,53-0,57, im Mittel 0,55. Die Scatchard-Anàlyse (8) ergibt bei Berücksichtigung der Eiweißbindung, welche die $K_{D}$, jedoch nicht $B_{\text {max. }}$ beeinflußt:

1. Ausdauertrainierte: $B_{\max .}=41,2 \mathrm{fmol} / 10^{7}$ Zellen ( $50 \%$ Vertrauensbereich $37-49,4 \mathrm{fmol} / 10^{7}$ Zellen), ungefähr 2500 Rezeptoren pro Zelle und $K_{D}=0,49$ nmol/l Dihydroalprenolol;
2. nicht Ausdauertrainierte: $B_{\max .}=21,6 \mathrm{fmol} / 10^{7}$ Zellen (50\% Vertrauensbereich $17,5-26,1 \mathrm{fmol} /$ $10^{7}$ Zellen), ungefähr 1300 Rezeptoren pro Zelle und $K_{D}=0,44 \mathrm{nmol} / 1$ Dihydroalprenolol (Abb. 2).

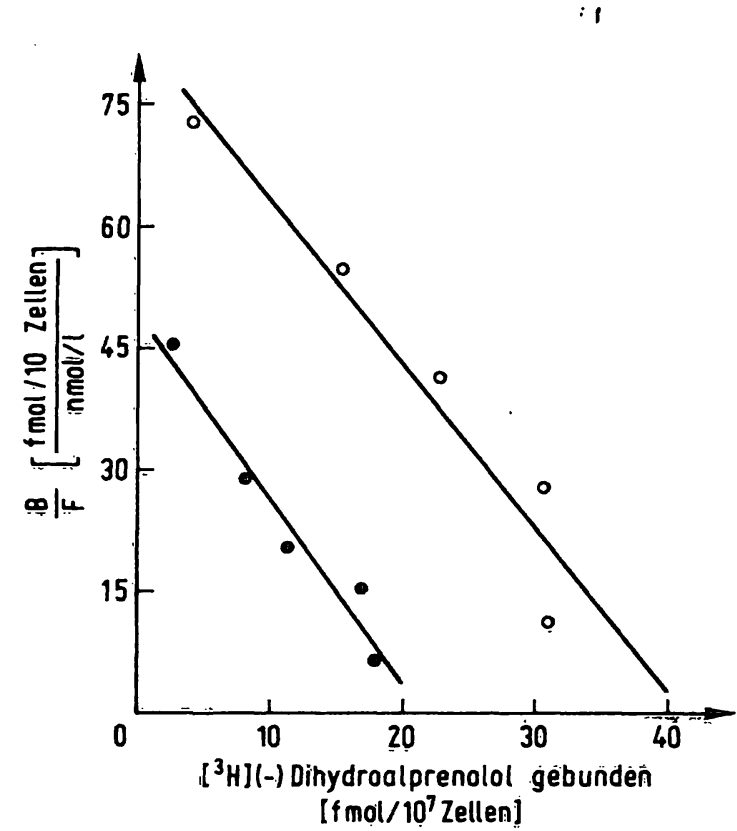

Abb. 2. Die Scatchard-Regression für die Trainierten $(\mathrm{O}-\mathrm{O}, \mathrm{r}$ $=0,96)$ und Untrainierten $(-\otimes, r=-0,97)$ verlaufen annähernd linear, was als Indikator einer homogenen Rezeptorpopulation gedeutet werden darf. $\mathbf{B}_{\max }$ beträgt $41,5 \mathrm{fmol} / 10^{7}$ Zellen (Trainierte) und $22 ; 9 \mathrm{fmol} / 10^{7}$ Zellen (Untrainierte), $K_{D}$ 0,90 nmol/ (Trianierte) und $0,84 \mathrm{nmol} / \mathrm{t}$ (Untrainierte) Dihydroalprenolol.

Die Zahl der Rezeptoren pro Zelle wurde nach der Gleichung

$\frac{\text { Rezeptoren }}{\text { Zelle }}=$

$\frac{\text { Dihydroalprenolol gebunden }}{\text { Zellzahl }} \cdot \frac{6,02 \cdot 10^{23} \text { Molèküle }}{\mathrm{mol}}$

berechnet. Die unspezifische Aufnahme von Dihydroalprenolol durch die Zellen verhält sich sowohl bei Trainierten ( $r=0,997$, Medianwerte der Doppelbestimmungen von 6 Probanden) wie Üntrainierten ( $r=0,997$, Medianwerte der Doppelbestimimungen von 9 Probanden) linear zur Konzentration von Dihydroalprenolol. Die spezifische Bindung nimmt für den beobachteten Bereich von ungefähr 500035000 Zellen pro Liter, entsprechend ungefähr 450000-3125000 Zellen pro Filter bei Trainierten $(r=0,92, p<0,01$, Mittelwerte aus Doppelbestimmungen von 6 Probanden) und Untrainierten ( $r=$ $0,82, \dot{p}<0,01$, Mittelwerte aus Doppelbestimmungen von 9 Probanden) linear mit der Zellzahl zu. 


\section{Diskussion}

Anlaß zur Modifikation der Methode zur Isolierung von polymorphkernigen Leukocyten und der Bindungsstudie zur Charakterisierung $\beta$-adrenerger $\mathrm{Re}$ zeptoren an intakten Zellen, wie sie von Böyum (4) bzw. Dulis \& Wilson (2) beschrieben wurden, war die Beobachtung, da $B$ die gewonnenen Zellen sowohl in der Zellsuspension nach der Isolierung als auch im Inkubationsmedium (Hepes-Hank'sche Lösungen unterschiedlicher Modifikationen) erheblich zur Aggregation neigen. Diese Aggregation beeinflußt die Bindungsstudie in nicht voraussehbarer Weise und verfälscht die Ergebnisse. Die Isolierung der polymorphkernigen Leukocyten folgt weitgehend der von Böyum (4) beschriebenen Technik. Nach der Dichte-Gradienten-Zentrifugation wird die Sedimentation zur Trennung von Erythrocyten und polymorphkernigen Leukocyten jedoch in Plasmasteril ${ }^{\circledR}$ (1 Teil Zellsuspension, 1 Teil Plasmasteril $\left.^{\circledR}\right)$, durchgeführt, da sonst aus quantitativen Gründen eine weitere Blutentnahme zur Gewinnung von autologem, unverdünnten Plasma erforderlich geworden wäre. Nach der Sedimentation werden die separierten Leukocyten nicht in einer Pufferlösung, sondern dreimal im autologen Plasma (1:4 verdünnt mit physiol. NaCl-Lösung) gewaschen (,Top"-Fraktion nach der Dichte-Gradienten-Zentrifugation). Nach diesem Vorgehen konnte weder makroskopisch noch lichtmikroskopisch eine Aggregatbildung von polymorphkernigen Leukocyten vor Beginn der Bindungsstudie mit Dihydroalprenolol festgestellt werden.

In Abänderung des von Dulis \& Wilson (2) vorgeschlagenen Mediums (,Hanks' balanced salt solution was prepared according to Grand Island Biological Co") zur Inkubation der polymorphkernigen Leukocyten für die Bindungsstudie, wurden $200 \mu \mathrm{l}$ Zellsuspension in $2000 \mu \mathrm{l}$ autologem Plasma (1:4 mit physiol. $\mathrm{NaCl}$ verdünnt) inkubiert. Einerseits konnte dadurch die Bildung von Zellaggregationen verhindert werden, andererseits vermögen individuelle Bestandteile des Plasmas, welche die Bindungsstudie beeinflusșen können, wirksam zu werden, d.h., das Inkubationsmilieu ist weitgehend individuellen physiologischen Bedingungen angenähert. Auf das lysosomotrophe Chloroquin zur Reduktion der unspezifischen Bindung von Dihydroalprenolol konnte in Ubereinstimmung mit Dulis \& Wilson (2) wie auch Bieger et al. (9) nicht verzichtet werden, da sonst die spezifische Bindung nur $0-20 \%$ der Gesamtbindung beträgt. Ein weiterer Unterschied gegenüber Dulis \& Wilson (2) beruht darauf, daß zur Verdrängung von Dihydroalprenolol aus seiner spezifischen Bindung nicht Propranolol oder Katechola- mine wie (-)Adrenalin, sondern (-)Timolol in Ubereinstimmung mit Baer \& Porzig (5) bzw. Porzig et al. (6) verwendet wurde. In der Konzentration von $10^{-6} \mathrm{~mol} / \mathrm{l}$ an (-)Timolol darf eine stereospezifische, komplette Verdrängung von Dihydroalprenolol aus seiner spezifischen Rezeptorbindung an der intakten Zelle angenommen werden (6). Insgesamt ist die Güte der Studien mit davon abhängig, daß Äquilibrieren der Inkubationslösung, Inkubation und Filtriervorgang zeitlich exakt standardisiert werden.

Die spezifische Bindung von Dihydroalprenolol an intakten polymorphkernigen Leukocyten zeigt in Ubereinstimmung mit Dulis \& Wilson (2), wie auch Bieger et al. (9) bei einer Dosis von ungefähr 2 nmol/1 Dihydroalprenolol Sättigungsverhalten. Dies wird auch von Porzig et al. (6) für Ratten-Reticulocyten und Meurs et al. (3) für intakte menschliche Lymphocyten berichtet. Die Analyse nach Scatchard (8) ergibt für die untersuchte spezifische Bindung von Dihydroalprenolol an intakten polymorphkernigen Leukocyten einen linearen Verlauf (Abb. 2), der als Indikator einer homogenen Rezeptorpopulation gedeutet werden darf. Entsprechend dieser Analyse kann die maximale spezifische Bindung an Dihydroalprenolol für die Ausdauertrainierten mit 41,2 fmol $/ 10^{7}$ Zellen und für die nicht Ausdauertrainierten auf $21,6 \mathrm{fmol} / 10^{7}$ Zellen berechnet werden. Hieraus ergibt sich eine $\beta$-Rezeptorenzahl von ungefähr 1300 pro Zelle (nicht Ausdauertrainierte) und 2500 pro Zelle (Ausdauertrainierte). Diese Resultate sprechen dafür, daß dynamisches körperliches Ausdauertraining ein mittelbarer Faktor zur Steigerung der $\beta$-Rezeptorenzahl an polymorphkernigen Leukocyten sein kann. Damit übereinstimmende Befunde wurden von Bieger et al. (9) berichtet. Eine unmittelbare Ursache zur Erhöhung der $\beta$-Rezeptorendichte kann die trainingsabhängige Reduktion freier, zirkulierender Katecholamine sein (10-13), die vom Organismus auf zellulärer Ebene mit einer Gegenregulation beantwortet wird. An Membranpräparationen von Lymphocyten wurden entgegengesetzte, trainingsabhängige Befunde von Butler et al. (14) berichtet. Die Ursache dieser widersprüchlichen Resultate ist unklar; möglicherweise waren Trainingsdauer und Intensität ineffektiv oder die Lymphocytenfraktion oder aber Subpopulationen dieser Fraktion zeigen ein anderes Verhalten als polymorphkernige Leukocyten. Der Unterschied kann auch darauf beruhen, daß Butler et al. (14) im Gegensatz zur vorliegenden Arbeit, die Bindungsstudien an Membranpräparationen durchführten, die an sich über weniger spezifische Bindungsstellen verfügen als intakte Zellen (3). 
An Hand der Scatchard-Analyse (Abb. 2) wurde die $\mathrm{K}_{\mathrm{D}}$ zur 0,44 (Untrainierte) und $0,49 \mathrm{nmol} / 1$ Dihydroalprenolol (Trainierte) berechnet. Sie ist etwas höher als an Ratten-Retikulocyten für Dihydroalprenolol ermittelt $(8,14)$ und liegt in der gleichen Größenordnung wie von Dulis \& Wilson (6) an polymorphkernigen Leukocyten bestimmt. Dulis \& Wilson (2) fanden an menschlichen polymorphkernigen Leukocyten, allerdings nur bei 3-4 Probanden, eine $\beta$-Rezeptorendichte von ungefähr 1700 pro Zelle; an intakten menschlichen Lymphocyten werden von Meurs et al. (3) ungefähr 1500-1700 $\beta$-Rezeptoren pro Zelle beschrieben. In beiden Untersuchungen findet sich jedoch keine Definition der untersuchten Probanden, so daß direkte Vergleiche schwierig sind. Das mit der vorliegenden Untersuchung beobachtete Gesamtkollektiv weist ungefähr $1800 \mathrm{Re}-$ zeptoren pro Zelle, die Untrainierten ungefähr 1300 pro Zelle, die Trainierten 2500 pro Zelle auf. Diese Unterschiede weisen auf die Notwendigkeit einer genauen Definition der zu untersuchenden Probanden hin.

Dulis \& Wilson (2) schlagen zur Hemmung der unspezifischen lysosomalen Aufnahme von Dihydroalprenolol durch intakte Zellen das lysosomotrope Chloroquin vor. In Anwesenheit von $50 \mu \mathrm{mol} / \mathrm{l}$ Chloroquin beträgt die spezifische Bindung von $\mathrm{Di}-$ hydroalprenolol $70 \%$ bei einer Doṣis von 0,36 nmol/1 Dihydroalprenolol der Gesamtbindung und $29 \%$ bei $5,1 \mathrm{nmol} / 1$ Dihydroalprenolol. Eine statistische Untersuchung wird allerdings nicht vorgelegt. Dies trifft auch zu für die Studien von Meurs et al. (3), welche durch das ebenfalls lysosomotrope Phentolamin in Anwesenheit von Propranolol die unspezifische Bindung (oder Aufnahme) von Dihydroalprenolol durch intakte Lymphocyten von $75 \%$ auf $45 \%$ bei einer Konzentration von 1,6 nmol/1 Dihydroalprenolol und $10^{-4} \mathrm{~mol} / 1$ Phentolamin reduzieren können. Mit der vorliegenden Untersuchung kann gezeigt werden, daß die spezifische Bindung von Dihydroalprenolol in Anwesenheit von 50 $\mu \mathrm{mol} / 1$ Chloroquin im autologen Plasma als Inkubationsmedium bei einer Konzentration von 0,1 bis 0,5 $\mathrm{nmol} / 1$ Dihydroalprenolol im Mittel (Median) über $80 \%$ und im Dosisbereich von 1,0 bis $2,0 \mathrm{nmol} / 1 \mathrm{Di}$ hydroalprenolol von $70 \%$ auf $50 \%$ abnimmt. Eine Ursache für die beobachtete, etwas höhere spezifische Bindung von Dihydroalprenolol gegenüber der in einer Pufferlösung $(2,3)$ beobachteten kanñ die Reinkubation der Leukocyten im autologen Plasma zur Durchführung der Bindungsstudie sein.

Die Frage der Úbertragbarkeit der vorliegenden Resultate an peripheren Blutzelleñ auf Organe wie z: B. das Heřż muß derzeit offen blẹiben. Hierżu sind simultane Bestimmungen zu fordern. Immerhin scheint aber die Reduktion $\beta$-adrenerger Bindungsstellen an Myokardzellen von Patienten mit Ruhe= herzinsuffizienz um den Faktor 3-4 (15) in der gleichen Größenordnung zu liegen, wie an polymorphkernigen Leukocyten (16), wạs fụ̈r die Vergleichbar: keit spricht.

Zusammenfassend darf gefolgert werden, daß Waschen und Reinkubieren der polymorphkernigen Leukocyten mit autologem Plasma die beobachtete Neigung zur Zell-Aggregation verhindert. In Añwēsenheit von $50 \mu \mathrm{mol} / 1$ Chloroquin kann die spezifische Bindung von Dihydroạlpreñolól an iñtakten polymorphkernigen Leukocyten dosisabhängig auf $50 \%$ bis $80 \%$ erhöht wèrden. Die Dichte an $\beta$-adrenergen Bindungsstellen kann durch dynamisches körperliches Ausdauertraining gesteigert werdèn. Ausdauertraining stellt einen physiologischen Faktor zur ,up regulation" der $\beta$-Rezeptorendichte an polymorphkernigen Leukocyten, somit der Empfindlichkeit gegenüber Katecholaminen auf zellulärer Ebene dar. Kennntnisse der Regulation der $\beta=\mathrm{Re}-$ zeptoren können das Verständnis der Modulation der Aktivität des Sympathicus erwẹitern.

\section{Literatur}

1. Motulsky, H. J. \& Insel, P. A. (1982) N. Engl: J. Med. 307, 18-29.

2. Dulis, B. H. \& Wilson, I. B. (1980) J. Biol. Chem. 255, 1043-1048.

3. Meurs, H., Van den Bogaard, W., Kauffman, H. F. \& Bruynzeel, P. L. B. (1982) Eur. J. Pharmacol. 85, 185-194.

4. Böyum, A. (1967) Scand. J. Clin. Lab. Invest. 97 (Suppl),

- 77-89.

5. Baer, M. \& Porzig, H. (1980) FEBS Lett. 111, 205-208.

6. Porzig, H., Baer, M. \& Chanton, C. (1981) Naunyn-Schmiedebergs Arch. Pharmácol. 317, 286-293.

7. Weder, J. H. \& Bickel, M. H. (1970) Z. Anal. Chem. 252, 253-255.

8. Scatchard, G. (1949) Ann. N.Y. Acad. Sci. 51, 660-672.

9. Bieger, W., Zittel, R., Zappe, H. \& Weicker, H. (1982) Dtsch. Z. Sportmed. 33, 249.

10. Hartley, L. H., Mason, J. W., Hogan, R. P., Jones, L. G., Kotchen, T. A., Mougey, E. H., Wherry, F. E., Pennington, L. L. \& Ricketts, X. Y. (1972) J. Appl. Physiol. 33, 602606.

11. Lehmann, M., Keul, J., Huber, G. \& Da Prada, M. (1981) Int._J. Sports Mẹd. 2, 143-147.

12. Péronnet, F., Cléroux, J., Perrault, H., Cousineau, D., Cham- plain, J. \& Nadeau, R. (1981) J. Appl. Physiol. 51, 812-815. 
13. Winder, W. W., Hickson, R. C., Hagberg. J. M., Ehsani, A. A. \& McLane, J. A. (1979) J. Appl. Physiol. 46, 766-771.

14. Butler, J., O'Brien, M., O'Malley, K. \& Kelle, J. G. (1982) Nature 298, 60-61.
15. Bristow, M. R., Ginsburg, R., Minobe, W., Cubicciotti, R. S., Sagemann, W. S., Lurie, K., Billingham, M., Harrison, D. C. \& Stinton, E. B. (1982) N. Engl. J. Med. 307, 205-211.

16. Lehmann, M., Rühle, K., Schmid, P., Klein, H., Matthys, K. \& Keul, J. (1983) Z. Kardiol. 72, 529-536.

Priv.-Doz. Dr. M. Lehmann

Med. Univ.-Klinik

Abt. Sport- u. Leistungsmedizin

Hugstetter Str. 55

D-7800 Freiburg 


;

$$
\text { (2) }
$$

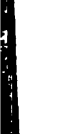

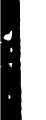

,

。 